\title{
On-orbit Performance and Calibration Improvements for the Reflective Solar Bands of Terra and Aqua MODIS
}

\author{
Amit Angal ${ }^{\mathrm{a}}$, Xiaoxiong (Jack) Xiong ${ }^{\mathrm{b}}$, Aisheng $\mathrm{Wu}^{\mathrm{a}}$, \\ Hongda Chen ${ }^{\mathrm{a}}$, Xu Geng ${ }^{\mathrm{a}}$, Daniel Link ${ }^{\mathrm{a}}$, Yonghong $\mathrm{Li}^{\mathrm{a}}$, Andrew Wald ${ }^{\mathrm{c}}$, and Jake Brinkmann ${ }^{\mathrm{a}}$ \\ ${ }^{a}$ Science Systems and Applications Inc. 10210 Greenbelt Rd, Lanham, MD 20706 \\ ${ }^{b}$ Sciences and Exploration Directorate, NASA/GSFC, Greenbelt, MD 20771 \\ ${ }^{c}$ Global Science and Technology Inc. 7855 Walker Drive, Suite 200 Greenbelt, Maryland 20770
}

\begin{abstract}
Moderate Resolution Imaging Spectroradiometer (MODIS) is the keystone instrument for NASA's EOS Terra and Aqua missions, designed to extend and improve heritage sensor measurements and data records of the land, oceans and atmosphere. The reflective solar bands (RSB) of MODIS covering wavelengths from $0.41 \mu \mathrm{m}$ to $2.2 \mu \mathrm{m}$, are calibrated on-orbit using a solar diffuser (SD), with its on-orbit bi-directional reflectance factor (BRF) changes tracked using a solar diffuser stability monitor (SDSM). MODIS is a scanning radiometer using a two-sided paddlewheel mirror to collect earth view (EV) data over a range of $\pm 55^{\circ}$ off instrument nadir. In addition to the solar calibration provided by the SD and SDSM system, lunar observations at nearly constant phase angles are regularly scheduled to monitor the RSB calibration stability. For both Terra and Aqua MODIS, the SD and lunar observations are used together to track the on-orbit changes of RSB response versus scan angle (RVS) as the SD and SV port are viewed at different angles of incidence (AOI) on the scan mirror. The MODIS Level 1B (L1B) Collection 6 (C6) algorithm incorporated several enhancements over its predecessor Collection 5 (C5) algorithm. A notable improvement was the use of the earth-view (EV) response trends from pseudo-invariant desert targets to characterize the on-orbit RVS for select RSB (Terra bands 1-4, 8, 9 and Aqua bands 8,9) and the time, AOI, and wavelengthdependent uncertainty. The MODIS Characterization Support Team (MCST) has been maintaining and enhancing the C6 algorithm since its first update in November, 2011 for Aqua MODIS, and February, 2012 for Terra MODIS. Several calibration improvements have been incorporated that include extending the EV-based RVS approach to other RSB, additional correction for SD degradation at SWIR wavelengths, and alternative approaches for on-orbit RVS characterization. In addition to the on-orbit performance of the MODIS RSB, this paper also discusses in detail the recent calibration improvements implemented in the MODIS L1B C6.
\end{abstract}

\section{Introduction}

The reflective solar bands (RSB) for Terra and Aqua MODIS instruments cover the 0.41 to $2.2 \mu \mathrm{m}$ wavelength range. Both MODIS instruments underwent extensive prelaunch characterization. The on-orbit calibration of the RSB is primarily based on solar diffuser (SD) and lunar measurements. In the case of some short wavelength bands, the on-board measurements are supplemented with earth-scene measurements from pseudo-invariant desert targets for a more accurate characterization of the non-linear scan-angle dependence. The design of the MODIS instrument and calibration mechanism has been well documented [1], [2], [3]. The goal of this paper is to present a detailed summary of the on-orbit calibration performance of the MODIS RSB. The MODIS Collection 6 (C6) Level 1B (L1B) algorithm has been operational since November, 2011 for Aqua MODIS, and February, 2012 for Terra MODIS and has enhanced the accuracy of the MODIS products in comparison with its predecessor Collection 5 (C5) L1B. Since then, several calibration improvements have been incorporated to further enhance the calibration accuracy.

Section 2 provides a brief review of MODIS RSB calibration and the main improvements in Collection 6 . The onorbit performance is assessed in section 3 with specific subsections detailing long-term performance of the SD degradation, gain, RVS, signal-to-noise ratio (SNR), uncertainty, as well as measurements of earth-view targets. Through data analysis and collaboration with the MODIS Sensor Working Group (MsWG), the MODIS Characterization Support Team (MCST) has identified a number of improvements to the C6 algorithm. These enhancements are detailed in section 4. Also discussed in section 4 are future plans for algorithm improvement and the associated challenges when calibrating these sensors. This paper concludes with a summary in section 5 . 


\section{RSB Calibration Overview}

MODIS RSB are calibrated independently for each spectral band, detector, subframe, and mirror side. Additionally, the RSB calibration includes response versus scan angle (RVS) that is needed to track the on-orbit gain changes at different scan-angles. Each measurement frame of the Earth View (EV) scene is recorded at a different angle of incidence on the rotating double-sided scan mirror. For most RSB, the RVS is based on measurements of the onboard $\mathrm{SD}\left[\mathrm{AOI}=50.25^{\circ}\right.$ ] and Moon $\left[\mathrm{AOI}=11.25^{\circ}\right.$. The SD calibration requires the SD door to be opened, so the SD can be illuminated by direct sunlight. A 7.8\% transmittance screen can be moved into place over the SD door to allow the calibration for high-gain ocean bands that would otherwise saturate. A spacecraft roll maneuver is scheduled approximately once a month to allow the instrument to measure the Moon through the instrument space view (SV) port [4].

The AOI-dependent gain is determined by a linear fit through these two data points over the entire AOI range of $10^{\circ}$ to $60.5^{\circ}$. For some of the shorter wavelength bands, a linear function is insufficient to accurately capture the RVS at AOIs far from the SD and SV port angles. The nonlinear effect is most apparent in MODIS bands 8 and 9 and also results in a long-term drift in the reflectance trending. MCST has developed an additional calibration procedure for these bands using EV data over pseudo-invariant calibration sites [PICS] [5]. These sites are located in North African desert regions that have demonstrated stable reflectance characteristics over mission timescales. MODIS views these site at several AOIs across the full AOI range. The instrument gain at all AOIs is modeled as a best-fit function of these earth-scene measurements along with the results from SD and lunar calibrations. The EV-based RVS has been implemented in Collection 6 Level 1B for Terra bands 1-4, 8-10 and Aqua bands 8 and 9[6]. As both missions continue to operate beyond their designed life, the EV-based RVS characterization approach could be extended to other bands.

\section{On-orbit Performance}

\section{A. SD degradation from SDSM measurement}

The SDSM is operated on a regular basis to monitor the degradation of the SD. In early mission, the SDSM was operated more frequently (weekly, bi-weekly) before its current configuration of once every three weeks. Due to an anomaly with the Terra SD screen (SDS) operation, the SD door has been fixed open with SDS permanently in place since July 2, 2003 (vertical dotted line in Figure 1a). This has resulted in the availability of an SD calibration every orbit, but the SDSM continues to be operated once every three weeks. In the case of Aqua, the SD door is still operable, a calibration with the SDS in place is performed once every three weeks and a calibration without the SDS is performed every six weeks.

Figure 1 shows the SD reflectance degradation trending for SDSM detectors $1(0.41 \mu \mathrm{m}), 4(0.55 \mu \mathrm{m})$, and $7(0.85$ $\mu \mathrm{m})$ of both MODIS instruments. A clear wavelength-dependent trend is evident with most degradation observed at short wavelengths, and with more degradation observed for Terra MODIS (due to increased exposure caused by the door anomaly). The results for other detectors further reaffirms the wavelength dependence with the D9 $(0.936 \mu \mathrm{m})$ exhibiting the least degradation. A summary of the total degradation with annual degradation rates is provided in Table I. More details of the performance of the SDSM can be found in [7]. 


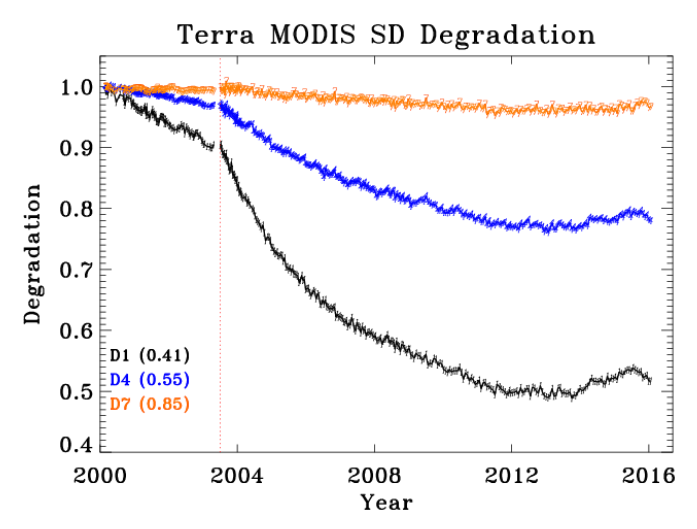

(a)

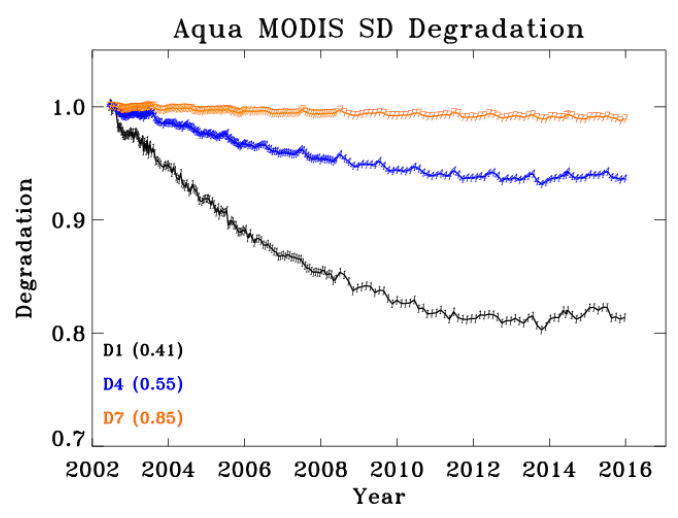

(b)

Figure 1. SD degradation using SDSM for (a) Terra MODIS and (b) Aqua MODIS.

Table I. Summary of the MODIS SD degradation

\begin{tabular}{|c|c|c|c|c|c|c|c|}
\hline & \multicolumn{4}{|c|}{ Terra MODIS } & \multicolumn{2}{c|}{ Aqua MODIS } \\
\hline $\begin{array}{c}\text { SDSM } \\
\text { detector }\end{array}$ & $\begin{array}{c}\text { Wavelength } \\
(\mu \mathrm{m})\end{array}$ & $\begin{array}{c}\text { Degradation I } \\
(1279 \text { days })\end{array}$ & $\begin{array}{c}\text { Annual } \\
\text { Rate(\%) }\end{array}$ & $\begin{array}{c}\text { Degradation } \\
\text { II }(5863 \\
\text { days })\end{array}$ & $\begin{array}{c}\text { Annual } \\
\text { Rate(\%) }\end{array}$ & $\begin{array}{c}\text { Degradation } \\
(5104 \text { days })\end{array}$ & $\begin{array}{c}\text { Annual } \\
\text { Rate }(\%)\end{array}$ \\
\hline D1 & 0.41 & 0.904 & 2.75 & 0.517 & 3.08 & 0.8137 & 1.33 \\
\hline D2 & 0.46 & 0.941 & 1.70 & 0.636 & 2.42 & 0.8778 & 0.87 \\
\hline D3 & 0.53 & 0.965 & 1.01 & 0.738 & 1.81 & 0.9244 & 0.54 \\
\hline D4 & 0.55 & 0.973 & 0.78 & 0.780 & 1.53 & 0.9367 & 0.45 \\
\hline D5 & 0.65 & 0.989 & 0.33 & 0.869 & 0.95 & 0.9646 & 0.25 \\
\hline D6 & 0.75 & 0.997 & 0.10 & 0.928 & 0.54 & 0.9812 & 0.13 \\
\hline D7 & 0.86 & 1.000 & 0.01 & 0.967 & 0.26 & 0.9894 & 0.08 \\
\hline D8 & 0.9 & 1.002 & -0.05 & 0.988 & 0.11 & 0.9922 & 0.06 \\
\hline D9 & 0.93 & 1.000 & -0.01 & 0.989 & 0.09 & 0.993 & 0.05 \\
\hline
\end{tabular}

\section{B. RSB gain trending:}

The gain trends for both instruments, derived from regular SD observations, are shown in Figure 2 for the visible wavelength bands $3(0.469 \mu \mathrm{m})$, band $8(0.412 \mu \mathrm{m})$ and band $9(0.443 \mu \mathrm{m})$. Figure 3 shows the corresponding gains derived from near-monthly lunar observations. The detector-averaged measurements are for mirror side 1 and are normalized to the first on-orbit measurement. Band 8 shows the largest change for Terra MODIS, up to 50\% from SD observations and $40 \%$ from lunar observations. Similarly, band 8 of Aqua MODIS shows the largest change among RSB, but with lunar gain change (40\%) exceeding the SD gain changes $(30 \%)$ over the mission lifetime.

While the Terra MODIS gain has a mirror side difference of up to $20 \%$ for band 8 , Aqua MODIS SD and lunar gain have a smaller mirror side difference ( $<5 \%$ for all bands). The NIR and SWIR band gains for Terra MODIS change less than $10 \%$ over the mission lifetime. In comparison, the Aqua MODIS NIR and SWIR band gains change less than 5\%. A comprehensive analysis of the MODIS RSB performance can be found in [1], [2], [3]. 

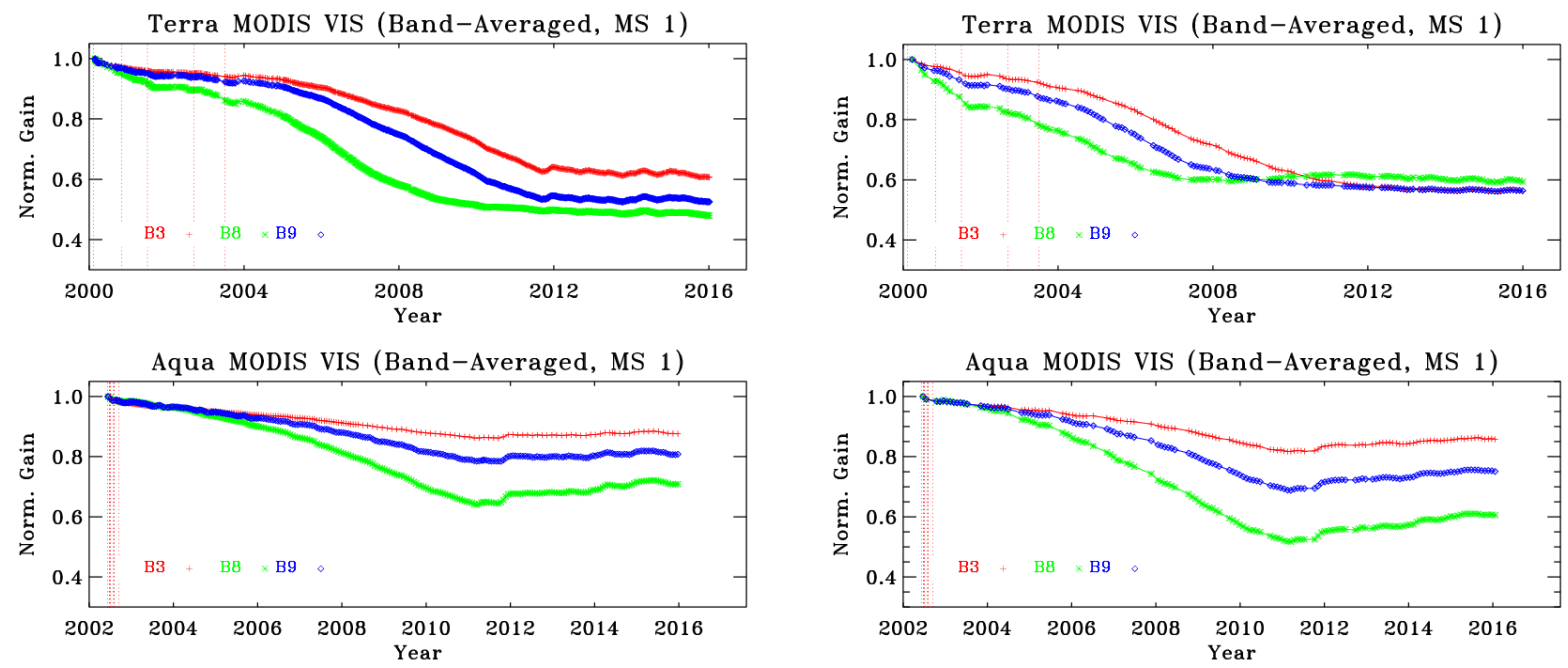

Figure 2. Terra and Aqua MODIS SD gain for bands 3, 8, Figure 3. Terra and Aqua MODIS lunar gain for bands 3, and 9.

8 , and 9.

\section{On-orbit Signal-to-Noise Ratio (SNR) Performance}

One of the key metrics used to assess the on-orbit performance is the SNR, which is monitored periodically using data from solar diffuser observations. A detailed description of the SNR calculation methodology and its on-orbit performance can be found in [8]. The on-orbit SNR is compared with the sensor-specified SNR to evaluate the inflight performance. With the exception of the short-wavelength VIS bands, which have experienced significant signal degradation, all other bands continue to meet the specification. Several noisy and inoperable detectors were identified in Aqua MODIS band 6 during prelaunch characterization. In addition, the SNR in Terra MODIS band 7 was observed to be below specification during prelaunch characterization and has continued to show the same behavior on-orbit. Table II shows the band-averaged SNR specification, prelaunch characterization, and on-orbit performance for years 2007 and 2015 for all RSB of both instruments. It is evident that the SNR for bands 3, 8 and 9 of both instruments does not meet the specification in recent years; however no significant impact is observed in the earth-scene measurements of these bands [8].

Table II. Summary of the MODIS on-orbit SNR performance

\begin{tabular}{|c|c|c|c|c|c|c|c|}
\hline & & \multicolumn{3}{|c|}{ Aqua MODIS } & \multicolumn{3}{c|}{ Terra MODIS } \\
\hline Band & Spec. & Prel & $\mathbf{2 0 0 7}$ & $\mathbf{2 0 1 5}$ & Prel & $\mathbf{2 0 0 7}$ & $\mathbf{2 0 1 5}$ \\
\hline 1 & 128 & 197 & 203 & 223 & 171 & 205 & 203 \\
\hline 2 & 201 & 525 & 527 & 569 & 418 & 506 & 512 \\
\hline 3 & 243 & 331 & 301 & 292 & 309 & 292 & 211 \\
\hline 4 & 228 & 333 & 321 & 325 & 309 & 308 & 256 \\
\hline 5 & 74 & 158 & 146 & 145 & 114 & 85 & 81 \\
\hline 6 & 275 & 114 & 474 & 487 & 394 & 391 & 375 \\
\hline 7 & 110 & 145 & 155 & 157 & 71 & 101 & 97 \\
\hline 8 & 880 & 1092 & 941 & 826 & 902 & 699 & 704 \\
\hline 9 & 838 & 1506 & 1423 & 1301 & 1147 & 1372 & 1053 \\
\hline 10 & 802 & 1528 & 1488 & 1466 & 1100 & 1482 & 1249 \\
\hline 11 & 754 & 1607 & 1729 & 1730 & 1152 & 1704 & 1540 \\
\hline 12 & 750 & 1436 & 1506 & 1496 & 983 & 1270 & 1071 \\
\hline
\end{tabular}

\begin{tabular}{|l|l|l|l|l|l|l|l|}
\hline & & \multicolumn{3}{|c|}{ Aqua MODIS } & \multicolumn{3}{c|}{ Terra MODIS } \\
\hline Band & Spec. & Prel & $\mathbf{2 0 0 7}$ & $\mathbf{2 0 1 5}$ & Prel & $\mathbf{2 0 0 7}$ & $\mathbf{2 0 1 5}$ \\
\hline 12 & 750 & 1436 & 1506 & 1496 & 983 & 1270 & 1071 \\
\hline $13 \mathrm{~L}$ & 910 & 1405 & 1472 & 1506 & 1173 & 1371 & 1375 \\
\hline $13 \mathrm{H}$ & 910 & 1551 & 1677 & 1758 & 1187 & 1428 & 1431 \\
\hline $14 \mathrm{~L}$ & 1087 & 1507 & 1595 & 1564 & 1315 & 1390 & 1265 \\
\hline $14 \mathrm{H}$ & 1087 & 1885 & 2017 & 1932 & 1337 & 1614 & 1477 \\
\hline 15 & 586 & 1519 & 1581 & 1623 & 754 & 1466 & 1438 \\
\hline 16 & 516 & 1380 & 1487 & 1480 & 713 & 1249 & 1250 \\
\hline 17 & 167 & 391 & 365 & 373 & 360 & 342 & 313 \\
\hline 18 & 57 & 93 & 91 & 95 & 92 & 91 & 94 \\
\hline 19 & 250 & 504 & 512 & 519 & 464 & 502 & 470 \\
\hline 26 & 150 & 229 & 283 & 285 & 212 & 249 & 237 \\
\hline
\end{tabular}




\section{RVS performance}

The RSB RVS was characterized prelaunch for both instruments and exhibited strong wavelength dependence, with larger RVS impact at short wavelengths. This is also true on-orbit, with band 8 (412 nm) showing the most RVS change for both instruments. Figures 4 and 5 show the on-orbit RVS change for mirror side 1 of band 8 plotted as a function of frame and time. At frame 17 (lunar frame), the on-orbit RVS change of band 8 mirror side 1 is as large as $34 \%$ for Terra and up to $20 \%$ for Aqua. The on-orbit RVS change of band 9 mirror side 1 can be as large as $16 \%$ for Terra and up to $12 \%$ for Aqua. Terra bands 3,10,11 and 12 show on-orbit RVS changes up to 13\%, 17\%, 12\%, and $9 \%$, respectively. For the remaining RSB, the on-orbit change is generally within 5\%.

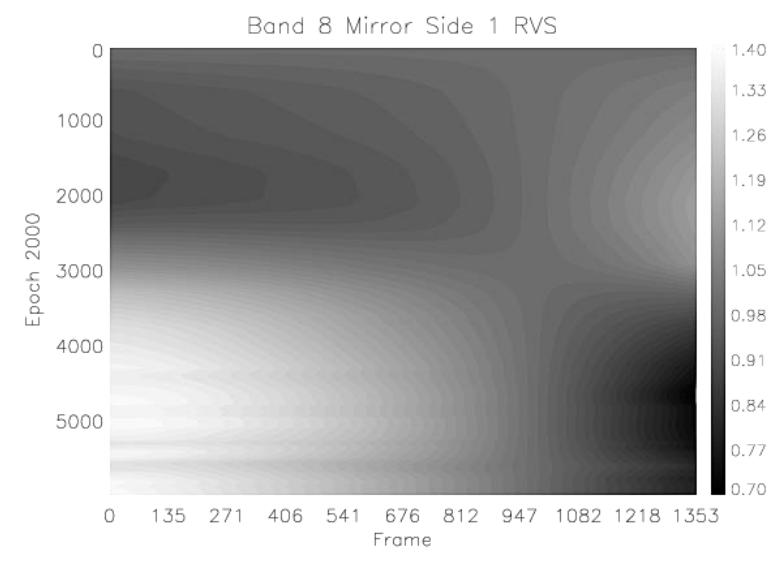

Figure 4: On-orbit RVS change for Terra band 8

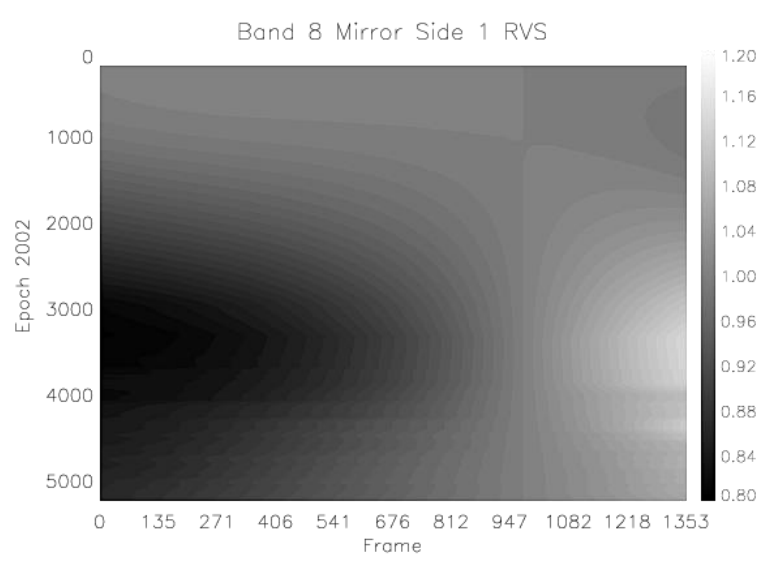

Figure 5: On-orbit RVS change for Aqua band 8

\section{E. RSB uncertainty}

The specified uncertainty for MODIS is $2 \%$ in reflectance and $5 \%$ in radiance at typical scene radiance levels and observations within a scan-angle range of $\pm 45^{\circ}$. An additional $1 \%$ uncertainty is assigned to observations made at other scan-angles and radiances out of the range from 0.3 specified typical radiance $(0.3$ Ltyp) to 0.9 specified maximum radiance $(0.9 \mathrm{Lmax})$. The L1B earth-view products include a pixel-level uncertainty index (UI) computed for every band. The coefficients are computed offline and represent the RSB calibration uncertainty that is based on measurements from prelaunch and on-orbit characterization [9].

The RSB uncertainty is determined by four independent look-up tables (known as u1, u2, u3, and u4). The u1 table is the combination of uncertainties from prelaunch BRF, SD screen vignetting function, earth-sun distance as well as the SD degradation and the on-orbit change in the SD response. The u2 table is updated regularly and incorporates the on-orbit changes associated with the RVS. The uncertainties from the on-board calibration measurements as well as earth-scene response are included in this term. The u3 table contains the uncertainty from the instrument temperature correction. The u4 table provides coefficients accounting for the scene-dependent SNR changes. An additional constant term u5 is designed to account for the uncertainty associated with electronic crosstalk and thermal leak observed in SWIR bands. Figures 6 and 7 show the percentage of total uncertainty (the quadrature sum of all five uncertainty contributions) of two representative years at typical scene radiance at nadir AOI for Terra and Aqua RSB, respectively. The SWIR bands are not included as they exhibit significant variation as a function of radiance levels. With the exception of Terra bands 3, 8, 9, and 10, most RSB continue to meet the specification of $2 \%$ for both years. In comparison, most Aqua MODIS RSB continue to meet the specification of $2 \%$. 


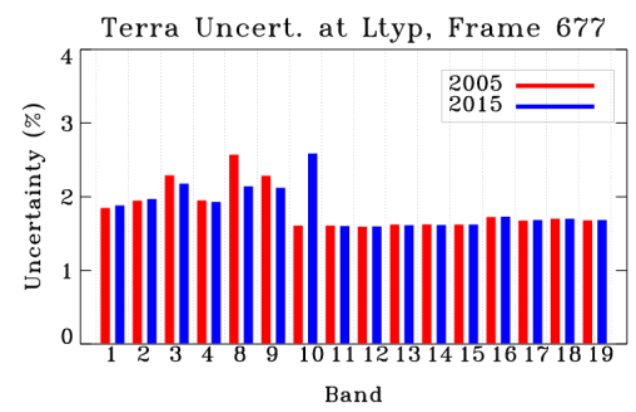

Figure 6. Terra RSB uncertainty

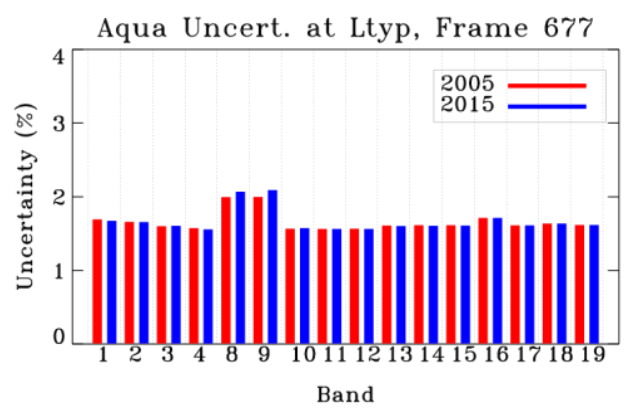

Figure 7. Aqua RSB uncertainty

\section{Calibration Improvements of Collection 6}

\section{A. Improvement in ocean bands calibration}

The EV-based RVS has been applied to Terra bands 1-4, 8 and 9 since the beginning of Collection 6 . For Terra MODIS band 10, long-term observations of the various pseudo-invariant desert sites revealed a long-term reflectance drift when using the standard on-board RVS calibration. The impacts of this drift were also evident in ocean color products. The EV-based RVS calibration is derived using observations of the high radiance Libyan desert sites, but these observations are saturated for Terra band 10. In order to overcome this limitation, an alternative approach using a lower-radiance Algerian desert site was developed. The EV-response trends from an Algerian site were used to characterize the sensor's behavior over the first six years and the Libyan sites (used for other bands) were used to track the changes thereafter. This approach significantly mitigated the long-term reflectance drift and improved the long-term accuracy of the band 10 calibration. Figure 8 shows the gain (m1/RVS) ratio between the on-board and earth-view based approaches. A long-term drift of up to $6 \%$ is observed at the SD AOI, and about $8 \%$ drift is observed at the nadir AOI. With the exceptions of some short term variations associated with forward LUT updates, the results at the lunar AOI from either approach agree to within $1 \%$. More details about this approach and its implementation can be found in [10]

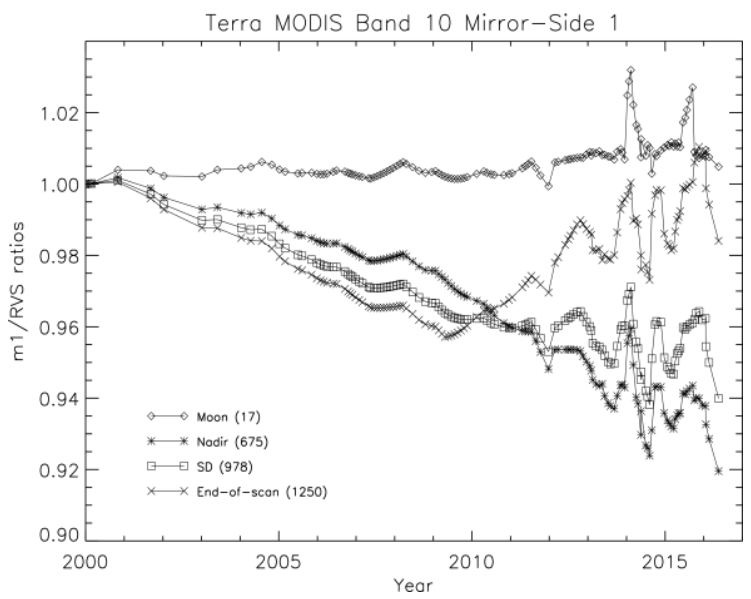

Figure 8. Terra MODIS Band 10 gain correction using EV-based RVS.

Due to the wavelength-dependent degradation observed in MODIS RSB, bands 11 and 12 are also expected to show long-term reflectance drift. However, due to the high gain setting of these bands, the desert observations are saturated for most of the mission. Using an unsaturated ocean target also proves difficult due to atmospheric contributions and low signal levels. In order to mitigate the impacts of the scene-dependence, MCST developed a ratio-approach to evaluate the long-term calibration consistency of bands 11 and 12 . A spectrally matching stable reference, band 4 is chosen to normalize the reflectance trends observed from these bands. Results indicate that the long-term drifts are within $2 \%$ for both bands. More testing is underway to further refine this methodology. 


\section{B. SWIR band degradation correction for SD}

The degradation of the MODIS SD is tracked using the SDSM over the 0.41 to $0.936 \mu \mathrm{m}$ spectral range. As shown in Table I, the SD degradation at $0.936 \mu \mathrm{m}$ is over $1 \%$ for Terra and about $0.7 \%$ for Aqua as of January 2016 . Since the SDSM cannot track any potential SD degradation beyond $0.94 \mu \mathrm{m}$, no correction of SD degradation is applied to the MODIS SWIR bands (5, 6, 7 and 26). Similar to the VIS and NIR bands, the SWIR bands are also monitored regularly using pseudo-invariant desert observations. Results indicate a long-term drift of up to $1.2 \%$ for Terra MODIS band 5. The likely cause behind this upward trend is the unaccounted degradation of the SD at wavelengths beyond $0.936 \mu \mathrm{m}$. Consequently, an EV-based correction was applied to Terra MODIS bands 5 and 26 to mitigate the impacts of this long-term drift. A similar correction is planned for Aqua band 5 as well as other SWIR bands when the reflectance trend shows a consistent bias of over $0.5 \%$ [11].

\section{Aqua Bands 1-4 calibration improvements}

In C6, the on-orbit RVS for Aqua bands 1-4 for all frames is derived solely based on the SD and lunar measurements using a linear fit through these measurements. While this approach has worked for most of the mission, reflectance degradation is observed in recent years, especially for bands 1 and 2. Consequently, the EVbased approach for on-orbit characterization of the RVS was extended to apply to bands $1-4$. Figures 9 and 10 show the normalized reflectance trending from the Libya 2 desert site for bands 1 and 2 of Aqua MODIS, respectively. Frames 501 and 649 represent the sensor's calibration performance around nadir. The blue symbols denote the topof-atmosphere reflectance derived from C6 and the red symbols denote the results from the EV-based approach. In both examples, the long-term reflectance drift observed in C6 products is mitigated using the EV-based RVS. The results of the revised algorithm are currently under review with the MODIS science team and an implementation in the forward processing is planned in the coming months.
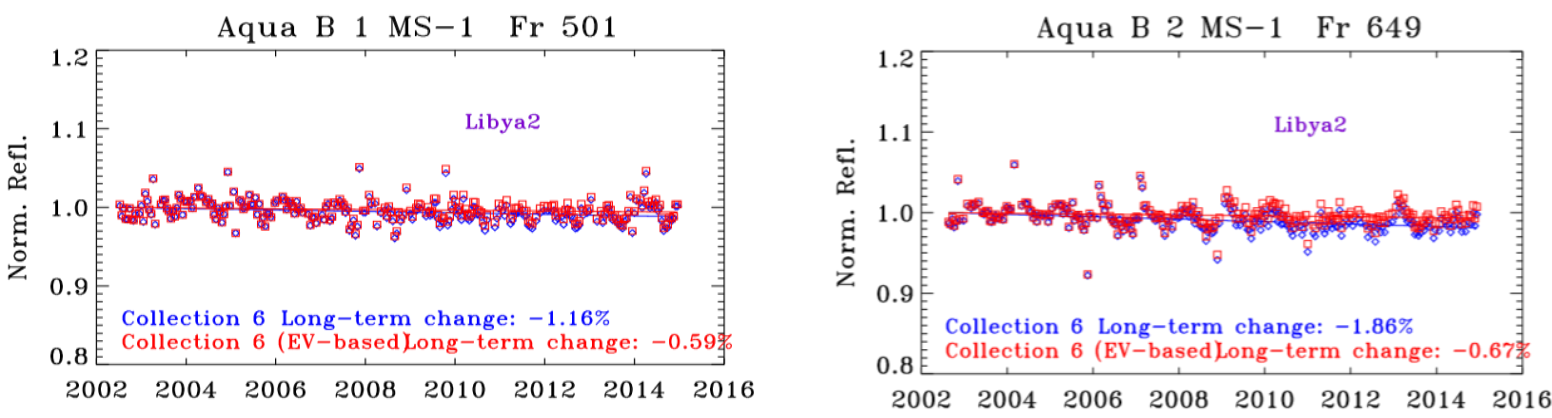

Figure 9: Aqua MODIS normalized reflectance at Libya 2 Figure 10: Aqua MODIS normalized reflectance at Libya PICS using band 1 , frame 501 .

2 PICS using band 2, frame 649.

\section{Progress with on-orbit polarization characterization}

During the prelaunch characterization, it was evident that the MODIS RSB are sensitive to the polarization of the incident light. Results indicate that the polarization sensitivity increases at large AOI and short wavelengths. As the Earth's top-of-atmosphere radiance becomes more polarized at shorter wavelengths due to Rayleigh scattering, initial polarization characterization is focused on short wavelengths bands 8,9 , and 3. On-orbit measurements show a significant change in the polarization sensitivity with a larger impact observed for Terra MODIS than for Aqua MODIS. The RSB calibration is based on measurements of weakly polarized light from the SD and the Moon. The effects of polarization sensitivity can be seen most strongly in the EV-response trends used for EV-based RVS. This sensitivity needs to be characterized precisely in terms of the polarization factor and phase angle to retrieve an accurate response [12], [13].

The impact of polarization on the response from the Libyan Desert sites is evaluated by estimating the top row of the instrument Mueller matrix based on simulated at-sensor response using Second Simulation of a Satellite Signal in the Solar Spectrum Vector (6SV), radiative transfer code [14]. Figures 11 and 12 show the normalized earth-view response trends for Terra MODIS band 8 at frames 790 and 1300, respectively. The symbols in black denote the background corrected, band-averaged response for mirror side 1 of band 8 and the red symbols are the polarization 
corrected response. As noted in the prelaunch characterization, the larger the frames, the more the sensitivity to polarization. The seasonal oscillations are more obvious after the year 2007.

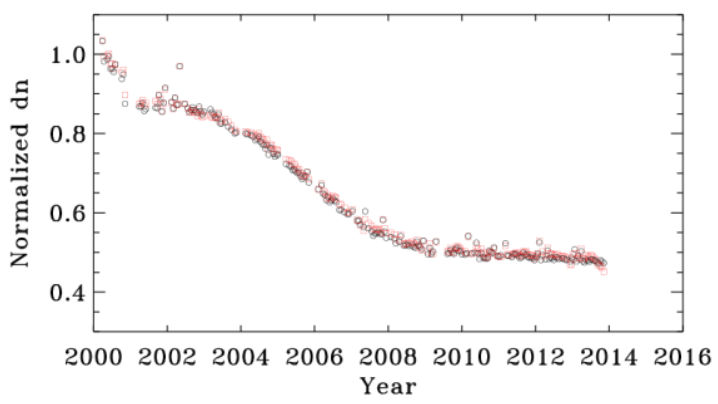

Figure 11. Terra MODIS band 8 EV-response trend atFigure 12. Terra MODIS band 8 EV-response trend at frame 790 .

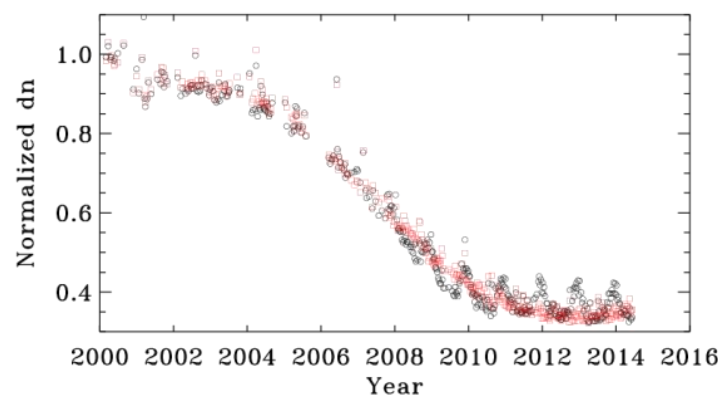

frame 1300.

\section{Summary}

MODIS is a key instrument on NASA's Terra and Aqua missions providing continuous measurements since launch. This paper has provided details of the on-orbit calibration of the MODIS RSB, their performance, and calibration algorithm enhancements incorporated in C6. The RSB calibration is reflectance-based, derived from regular measurements from the SD and Moon. Due to the degradation observed in the short-wavelength RSB, supplemental information from the pseudo-invariant EV observation is used to derive the on-orbit RVS. The SD degradation continues to be monitored by regular SDSM calibrations with the largest gain changes measured for shorter wavelengths. On-orbit gain changes for both Terra and Aqua MODIS are also most significant at shorter wavelengths. Terra band 8 has the largest change of nearly 50\% compared with the first on-orbit measurement. The C6 L1B algorithm incorporated several improvements over its previous versions. As the aging instruments continue to operate beyond their design lifetime several calibration improvements have been incorporated accordingly. The EV-based RVS has been extended to apply to Terra band 10 therefore resulting in an improvement in the long-term drift by about 6\%. The correction for the SD degradation for SWIR bands (wavelengths $1.24 \mu \mathrm{m}$ and $1.3 \mu \mathrm{m}$ ) has been incorporated in the C6 for Terra MODIS. Similar correction will be implemented as needed for other SWIR bands. The EV-based RVS algorithm has also been extended to Aqua bands 1-4 resulting in an improved on-orbit RVS characterization. Preliminary corrections for changes in polarization sensitivity will be implemented with a more comprehensive testing planned in the coming months.

Acknowledgement: The authors would like to thank the contributions of previous MCST members, especially Junqiang Sun for his contribution to MODIS RSB calibration and C6 development. The authors would also like to thank Kevin Twedt for a technical review of this manuscript.

\section{References}

[1] Xiong, X., M.. D. King, V.. Salomonson, W. Barnes, B. N. Wenny, A. Angal, A. Wu, S. Madhavan, and D. Link, "Moderate Resolution Imaging Spectroradiometer on Terra and Aqua Missions", John Wiley \& Sons, Ltd, vol. 9781118945179, pp. 53-89, 2015.

[2] Xiong, X., J. Sun, W. Barnes, V. Salomonson, J. Esposito, H. Erives, and B. Guenther, "Multiyear On-Orbit Calibration and Performance of Terra MODIS Reflective Solar Bands", IEEE Trans. Geosci. Remote Sens., vol. 45, issue 4, pp. 879-889, 2007.

[3] Xiong, X., J. Sun, X. Xie, W. Barnes, and V. Salomonson, "On-Orbit Calibration and Performance of Aqua MODIS Reflective Solar Bands", IEEE Trans. Geosci. Remote Sens., vol. 48, issue 1, pp. 535-546, 2010.

[4] Sun, J., X. Xiong, W. Barnes, and B. Guenther, "MODIS Reflective Solar Bands On-Orbit Lunar Calibration", IEEE Trans. Geosci. Remote Sens., vol. 45, issue 7, pp. 2383-2393, 2007. 
[5] Sun, J., X. Xiong, A. Angal, H. Chen, A. Wu, and X. Geng, "Time-Dependent Response Versus Scan Angle for MODIS Reflective Solar Bands", IEEE Transactions on Geoscience and Remote Sensing, vol. 52, issue 6, pp. 31593174, 2014.

[6] Toller, G., X. Xiong, J. Sun, B. N. Wenny, X. Geng, J. Kuyper, A. Angal, H. Chen, S. Madhavan, and A. Wu, "Terra and Aqua Moderate-resolution Imaging Spectroradiometer Collection 6 Level 1B Algorithm", Journal of Applied Remote Sensing, vol. 7, issue 1, 2013.

[7] Xiong, X., A. Angal, J. Sun, J. Choi, and E. Johnson, "On-orbit performance of MODIS Solar Diffuser Stability Monitor", Journal of Applied Remote Sensing, vol. 8, pp. 083514-1-14, 2014.

[8] Angal, A., X. Xiong, J. Sun, and X. Geng, "On-orbit noise characterization of MODIS reflective solar bands", J. Appl. Remote Sens. 0001;9(1):094092, 2015.

[9] Xiong, X., J. Sun, A. Wu, J. Esposito, and W. Barnes, "Terra and Aqua MODIS calibration algorithms and uncertainty analysis, Sensors, Systems, and Next-Generation Satellites IX", Proc. SPIE, vol. 5978, no. 59780V, 2005.

[10] Geng, X., A. Angal, J. Sun, H. Chen, A. Wu, Y. Li, D. Link, and X. Xiong, "Status of time-dependent response versus scan-angle (RVS) for Terra and Aqua MODIS reflective solar bands", Proc. SPIE 9218, Earth Observing Systems XIX, 92181P, 2014.

[11] Angal, A., A. Wu, X. Xiong, X. Geng, D. Link, and H. Chen, "On-orbit performance of the MODIS SWIR bands", Proc. SPIE 9264, Earth Observing Missions and Sensors: Development, Implementation, and Characterization III, 92641Q, 2014.

[12] Sun, J., and X. Xiong, "MODIS Polarization Sensitivity Analysis", IEEE Trans. Geosci. Remote Sens., vol. 45, no. 9, pp. 2875-2885, 2007.

[13] Lyapustin, A., Y. Wang, X. Xiong, G. Meister, S. Platnick, R. Levy, B. A. Franz, S. Korkin, T. Hilker, J. Tucker, et al., "Scientific impact of MODIS C5 calibration degradation and C6 improvements", Atmospheric Measurement Techniques Discussions, vol. 7, issue 7, pp. pp.7281-7319, 2014.

[14] Wu, A., X. Geng, A.E.Wald, and X. Xiong, "Tracking Terra MODIS on-orbit polarization sensitivity using pseudo-invariant desert sites ", Proc. SPIE 9639, Sensors, Systems, and Next-Generation Satellites XIX, 963914, 2015. 